\title{
Ectopic thymoma simulating a pericardial cyst
}

\author{
G. Caramori1, N. Calia1, C. Pasquini1, O. Arar1, F. Ravenna1, I. Guzzinati1, \\ A. Boniotti1, L. Cavazzini3, A. Ciaccia1, G. Cavallesco2, A. Papi1
}

ABSTRACT: Ectopic thymoma simulating a pericardial cyst. G. Caramori, N. Calia, C. Pasquini, O. Arar, F. Ravenna, I. Guzzinati, A. Boniotti, L. Cavazzini, A. Ciaccia, G. Cavallesco, A. Papi.

We present a case of a 53 year old man with a thymoma near the pericardium, a rare ectopic localisation of thymoma. A round radiodensity found at the right cardiophrenic angle was initially suspected at the echocardiography to be a pericardial cyst. The diagnosis of thymoma was made only after histopathological examination of the surgically re-sected lesion.

Monaldi Arch Chest Dis 2005; 63: 4, 230-233.

Keywords: Ectopic thymoma, pericardial mass, chest imaging.

${ }^{1}$ Dipartimento di Medicina Clinica e Sperimentale, Sezione di Malattie dell'Apparato Respiratorio and

2 Sezione di Chirurgia Toracica,

3 Dipartimento di Medicina Sperimentale e Diagnostica, Sezione di Anatomia, Istologia e Citologia Patologica, University of Ferrara, Italy.

Correspondence: Gaetano Caramori MD, PhD, Centro di Ricerca su Asma e BPCO, Università di Ferrara, Via Savonarola 9 , 44100 Ferrara, Italy; e-mail: crm@unife.it

This work has been funded by Azienda Ospedaliera Universitaria Arcispedale S. Anna di Ferrara and Fondazione Carife (Ferrara, Italy).

\section{Introduction}

Thymoma is a rare neoplasm but is the most common tumour of the anterior mediastinum accounting for $50 \%$ of anterior mediastinal masses and $15 \%$ of all mediastinal tumours [1,2]. The majority of thymomas are found in the normal location of the thymus; ectopic occurrence of thymoma is unusual $[1,2]$. Here we report a case of thymoma close to the pericardium in an adult.

\section{Case report}

A 53 year old man attended our clinic complaining of a two month history of dry cough and rhinitis. He denied recent fever and/or chills. The patient had always worked as a bank employee. The patient was a current smoker and had smoked a mean of twenty cigarettes a day for 20 years. He had been in good health until recently with no previous hospital admissions. His physical examination was unremarkable. A chest $\mathrm{x}$-ray was suggestive for the presence of a pericardial cyst (4 by 6 $\mathrm{cm}$ ) at the right cardiophrenic angle (fig. 1). Routine laboratory tests demonstrated only a hypochromic microcytic anemia (haemoglobin $11.4 \mathrm{~g} / \mathrm{dl}$, mean corpuscular volume $65 \mathrm{fL}$, mean corpuscular haemoglobin $21 \mathrm{pg}$ ), related to his beta thalassemic trait. Renal and liver function tests were normal. Forced vital capacity (FVC) $(105 \%$ of predicted value) and forced expiratory volume in one second $\left(\mathrm{FEV}_{1}\right)(110 \%$ of predicted value $)$ were normal. Echocardiography was suggestive for the presence of a pericardial cyst (5.9 by 3.27 $\mathrm{cm}$ ) near the right atrium. It also demonstrated the presence of mild tricuspid valve regurgitation and a systolic pulmonary arterial pressure of $37 \mathrm{mmHg}$ was estimated. A fibreoptic bronchoscopy was unremarkable. The bronchial lavage showed only an increased number of neutrophil granulocytes and squamous metaplasia. A chest computed tomographic (CT) scan, obtained with the administration of contrast material, revealed the presence of a round, well-defined, mass $(7.5$ by $7.7 \mathrm{~cm})$ in the right paracardiac area, with density of a solid lesion and evident contrast enhancement (fig. 1). Lymphadenomegaly (less of $1 \mathrm{~cm}$ in diameter) was present in the Barety's region. A CT scan of the abdomen was normal. He was therefore referred for surgical resection of the lesion using video assisted thoracic surgery. Macroscopically the lesion was extrapericardial, located in the right paracardiac area, and invading the fatty tissue near the pericardium. A peduncle was connecting the lesion with the thymus remnant in the anterosuperior mediastinum.

On pathological examination the lesion was a large $(9,5$ by $7 \mathrm{~cm})$ round, hard mass with many smaller white, hard, nodules on the cut surface. Microscopic examination showed it to be a spindle type AB thymoma with abundant T-lymphocytes (fig. 2). There was absence of microscopic 


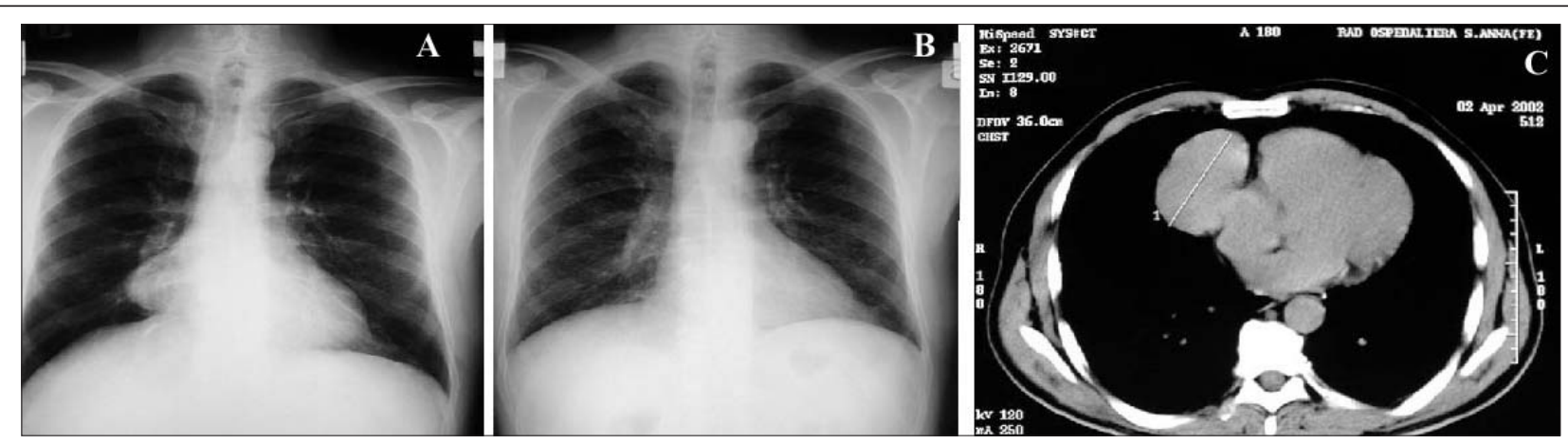

Fig. 1. - Chest-X-ray obtained before (A) and after (B) surgical resection, showing the disappearance (B) of the round radiodensity at the right cardiophrenic angle (A). (C) Computed tomographic scan of the chest obtained before surgical resection demonstrating a round, well-defined, mass $(7.5$ by $7.7 \mathrm{~cm})$ in the right paracardiac area, with density of a solid lesion and evident contrast enhancement.

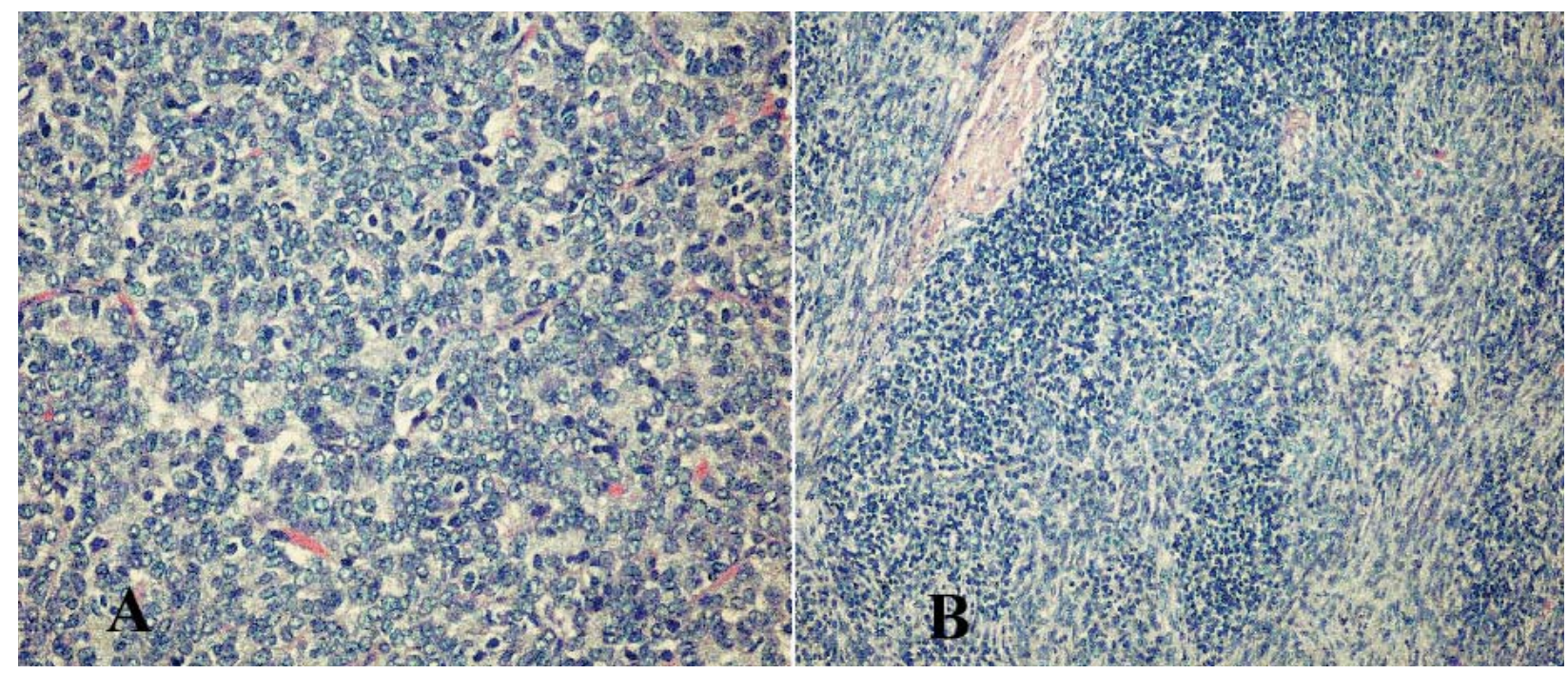

Fig. 2. - Microscopic findings: (A) spindle neoplastic epithelial cells (original magnification x150). (B) T-lymphocytes infiltrating the spindle neoplastic epithelial cells (original magnification x150).

capsular invasion (stage IIa according to Masaoka) [3].

He made an uneventful postoperative recovery. A postoperative echocardiography, a month later, and a chest x-ray, one year later, were both negative (fig. 1). After two years of follow-up there is complete absence of local recurrence and/or metastasis.

\section{Discussion}

A thymoma is a neoplastic proliferation of the epithelial cells of the thymus gland [1-4]. Thymomas may present at any age but are typically neoplasm of older adults (with a median age of 50 years), without gender preferences. They are uncommon in children [1]. The majority of thymomas are found in the normal location of the thymus in the anterosuperior mediastinum [1]. Ectopic occurrence of thymoma, has been rarely described in many other sites (e.g. neck, trachea, thyroid, lung, pleura) including the pericardium, sometimes associated with thymic rests in these tissues [2]. In such cases the alternative possibility of solitary metastases from a small undetected primary tumour in the thymus should always be considered. Ectopic thymomas are identical to those that arise in the normal location of the thymus but usually are not so clearly encapsulated [1].

Embriologically the thymus derives from the third, and to a lesser extent, the fourth pharyngeal pouches. During the sixth week of gestation, the endodermal lining of the ventral wing of the third pharyngeal pouch forms a pronounced sacculation, which subsequently detaches from the pharyngeal wall, giving rise to thymic primordia [5]. As development continues, the thymic primordia migrate in a caudal and medial direction along with the lower parathyroid glands. During the eighth week, these primordia enlarge toward their lower ends, forming two thymic lobes that fuse along the midline to occupy their definitive position within the anterosuperior mediastinum [5]. Usually, during this descent, the cranial portion of the two thymic lobes breaks up into small fragments that usually disappear but that may persist in the soft tissues of the neck, often in intimate connection with the lower parathyroid gland and sometimes embed in the thyroid [5]. The third and fourth pharyngeal pouches are located strictly adjacent to the pericardial sac, for this reason sometimes thymic rests may migrate with the latter into the middle and lower mediastinum.

In 1999, a World Health Organisation (WHO) consensus committee has published the histologic 
criteria for the diagnosis of thymic epithelial tumours (thymomas, thymic carcinomas, thymic carcinoids) [4]. Thymic epithelial tumours are divided in types A, B, AB and $\mathrm{C}$. The letter " $\mathrm{A}$ " corresponds to "atrophic", mimicking the adult thymus, "B" indicates "bioreactive", resembling the biologically active organ in the fetus and infant and "C" denotes "carcinoma" [4]. Type A thymomas comprise a homogenous population of neoplastic epithelial cells with spindle/oval shape, lacking nuclear atypia, and accompanied by few or no non-neoplastic lymphocytes. Type B thymomas have foci with the features of type A thymoma admixed with foci rich in lymphocytes: the segregation of two patterns can be sharp or indistinct. Type B tumours are subdivided further into three subtypes designed B1, B2, and B3 respectively, on the basis of the proportional increase of the neoplastic epithelial component and the emergence of atypia of the neoplastic cells. Thymomas of type $\mathrm{AB}$ are tumours in which foci having the features of type A thymoma are admixed with foci rich in lymphocytes, with sharp or indistinct segregation of the two patterns. All kinds of thymic carcinomas are categorised as type C [4]. The neoplastic epithelial cells always contain cytokeratins and frequently express CD57. In around $70 \%$ of the cases there is also a population of non-neoplastic immature (cortical) T lymphocytes, which usually express terminal deoxynucleotidyl tranferase (TdT), CD1a, CD99, CD3, CD4, and CD8 $[1,4]$.

Most patients with thymomas are asymptomatic. In some patients the mass effect of the thymoma may cause cough, chest pain, dyspnea, dysphagia, hoarseness and the superior vena cava syndrome. Occasionally there is weight loss and/or unexplained fever [6]. Up to $40 \%$ of patients with thymomas have an autoimmune/paraneoplastic syndrome. Myasthenia gravis is the most frequent of these syndromes followed by pure red cell aplasia, and hypogammaglobulinemia, systemic lupus erythematosus and rheumatoid arthritis are uncommon [6].

The chest radiography shows a radiodense mediastinal mass. CT scan of the chest confirms the location and may reveal some characteristics of the thymic epithelial tumours (cystic changes or involvement of surrounding structures). $\mathrm{CT}$ is of limited value in differentiating type $\mathrm{AB}, \mathrm{B} 1, \mathrm{~B} 2$, and B3 thymomas. However, smooth contours and a round shape are most suggestive of type A thymomas, whereas irregular contours are most suggestive of type $C$ thymomas. Calcification is suggestive of type $\mathrm{B}$ thymomas [7].

The most appropriate diagnostic and therapeutic approach for a mediastinal mass is a complete surgical resection. In fact the exclusion of malignancy requires complete surgical resection of the lesion and its pathological examination. Serum tumour markers or immunophenotype analysis of blood lymphocytes are not useful in the diagnosis of thymoma.

The WHO histopathological classification has prognostic value. In a large series from Japan, the

\begin{tabular}{ll} 
Table 1. - Staging of Thymomas According to Masaoka \\
\hline Stage
\end{tabular} $\begin{array}{ll}\text { I } & \begin{array}{l}\text { Macroscopically and microscopically completely } \\
\text { encapsulated }\end{array} \\
\text { IIa } & \begin{array}{l}\text { Macroscopic invasion into surrounding fat or } \\
\text { pleura; }\end{array} \\
\text { IIb } & \begin{array}{l}\text { Microscopic invasion of capsule } \\
\text { III }\end{array} \\
\text { IVa } & \begin{array}{l}\text { Macroscopic invasion of adjacent organ } \\
\text { IVb }\end{array}\end{array}$

Data from [3].

20-year survival rates decrease from $100 \%$ (type A) to $87 \%$ for $\mathrm{AB}$ thymomas until $36 \%$ for $\mathrm{B} 3$ thymomas [4]. The most important indicator of long-term survival in thymomas is perhaps completeness of resection and this is closely associated to its stage. The most widely accepted staging system for thymoma is based on Masaoka's criteria (table 1) and has been shown to correlate with surgical resectability and long-term survival [3, 4]. As a matter of fact, the 20-year survival rates for thymomas decrease from around $90 \%$ for stages I and II, to $49 \%$ for stage III and $0 \%$ to stage IV [3].

The efficacy of adjuvant chemotherapy/radiotherapy for patients with stage III or IV thymoma remains controversial $[4,9]$.

Our patient had a stage IIa, AB thymoma, localised in an ectopic area, near the pericardium. The round radiodensity in the pericardial region seen on chest X-ray, was initially suspected to be a pericardial cyst on the basis of its radiologic and echocardiographic characteristics. Pericardial cysts are generally described as round radiodensities typically found at the right cardiophrenic angle in asymptomatic individuals [10]. Two-dimensional echocardiography and transesophageal echocardiography are usually extremely valuable in diagnosing the presence of a pericardial cyst [11]. However, in the case described the echocardiographic characteristics were mistakenly indicative of a pericardial cyst. At the initial visit, our patient presented only with a three month history of a dry cough and throughout the course of treatment and follow-up did not show any autoimmune/paraneoplastic syndrome. In ectopic thymomas, myasthenia gravis is in fact uncommon [6]. Pericardial thymoma may cause a "pseudocardiomegaly" on chest X-ray or pericardial effusion/tamponade [1214]. However, all these clinical features were absent in our patient.

In conclusion, this case illustrates the difficulty in establishing a correct diagnosis of pericardial cyst using chest $\mathrm{X}$-ray and echocardiography. It also suggests that any mediastinal mass noted on chest X-ray must be investigated, as early as possible, with a CT scan in order to detect the presence of potentially resectable neoplasms at a curable stage. 


\section{References}

1. Corrin B. Pathology of the lungs. Churchill Livingstone, London, UK, 2000: 521- 522.

2. Goldstraw P. Mediastinal masses. In: Gibson GJ, Geddes DM, Costabel U, Sterk PJ, Corrin B (Eds). Respiratory Medicine. 3rd ed, London, Elsevier Science Limited 2003: 1938-1958.

3. Masaoka A, Monden Y, Nakahara K, Tanioka T. Follow-up study of thymomas with special reference to their clinical stages. Cancer 1981; 48: 2485-2492.

4. Okumura M, Ohta M, Tateyama H, et al. The World Health Organization histologic classification system reflects the oncologic behavior of thymoma: a clinical study of 273 patients. Cancer 2002; 94: 624-632.

5. Suster S, Rosai J. Thymus. In: Sternberg SS, ed. Histology for pathologists. 2nd ed, Philadelphia, PA: Lippincott-Raven Publishers, 1997: 687-706.

6. Morgenthaler T, Brown L, Colby T, Harper C, Coles D. Thymoma. Mayo Clin Proc 1993; 68: 1110-23.

7. Tomiyama N, Johkoh $\mathrm{T}$, Mihara $\mathrm{N}$, et al. Using the World Health Organization Classification of thymic ep- ithelial neoplasms to describe CT findings. $A m J$ Roentgenol 2002; 179: 881-886.

8. Kondo K, Monden Y. Therapy for thymic epithelial tumors: a clinical study of 1,320 patients from Japan. Ann Thorac Surg 2003; 76: 878-884; discussion 884-885.

9. Zhu G, He S, Fu X, Jiang G, Liu T. Radiotherapy and prognostic factors for thymoma: A retrospective study of 175 patients. Int J Radiat Oncol Biol Phys 2004; 60: 1113-1139.

10. Feigin DS, Fenoglio JJ, McAllister HA, Madewell JE. Pericardial cysts. A radiologic-pathologic correlation and review. Radiology 1977; 125: 15-20.

11. Patel J, Park C, Michaels J, Rosen S, Kort S. Pericardial cyst: case reports and a literature review. Echocardiography 2004; 21: 269-272.

12. Ben Hami B, Caulet-Maugendre S, Valla J, HeresbachLe Berre N, Kernec J, Laurent M, Ramee MP. Primary pericardial thymoma: an unusual etiology of neoplastic pericarditis. Ann Pathol 1996; 16: 445-448.

13. Lanna TV, Sadaniantz A. Benign thymoma presenting as symptomatic pericardial tamponade. J Am Soc Echocardiogr 1999; 12: 679-681.

14. Sidhu US, Malhotra V, Chhina GS. An unusual case of pseudocardiomegaly. Chest 1998; 113: 1711-1714.

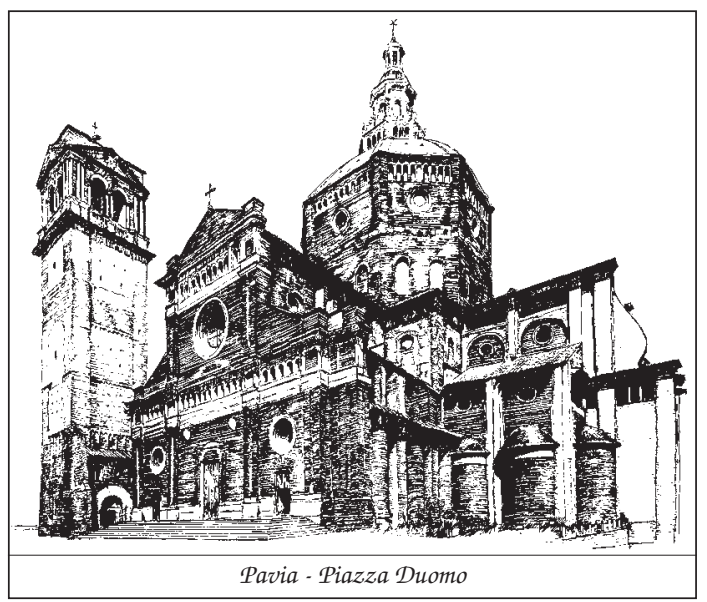

